\title{
An Association between Decreased Estimated Glomerular Filtration Rate and Arterial Stiffness
}

\author{
Ryuichi Kawamoto ${ }^{1}$, Katsuhiko Kohara ${ }^{2}$, Yasuharu Tabara ${ }^{2}$, Tetsuro Miki ${ }^{2}$, \\ Nobuyuki Ohtsuka ${ }^{1}$, Tomo Kusunoki ${ }^{1}$ and Nobukazu Yorimitsu ${ }^{1}$
}

\begin{abstract}
Objective Chronic kidney disease (CKD) is a major public health problem, but there is controversy over whether or not CKD is an independent risk factor for peripheral arterial stiffness in community residents.

Patients and Methods We randomly recruited 107 men, aged 68 \pm 9 (mean \pm standard deviation) years, and 203 women, aged $67 \pm 7$ years during their annual health examination in a single community. Study subjects did not have a clinical history of cerebrovascular disease or current neurological abnormalities. Peripheral arterial stiffness was evaluated by mean pulse wave velocity (PWV) determined at three points: from heart to the carotid artery, to the brachial artery, and to the ankle, and CKD was evaluated by estimated glomerular filtration rate (eGFR) using the Modification of Diet in Renal Disease Study Group equation.

Results Estimated GFR was significantly correlated with mean PWV ( $\mathrm{r}=-0.317, \mathrm{p}<0.001)$. Stepwise multiple linear regression analysis using mean PWV as an objective variable, adjusted by explanatory variables, showed that eGFR $(\beta,-0.171 ; \mathrm{p}<0.001)$ significantly contributed to mean PWV, along with age, body mass index, systolic blood pressure, diastolic blood pressure, and antihypertensive drug use, and improved multiple coefficient of determination in the model.
\end{abstract}

Conclusion Decreased eGFR is associated with an increased risk of arterial stiffness in community residents.

Key words: risk factor, atherosclerosis, renal function, eGFR, CKD

(Inter Med 47: 593-598, 2008)

(DOI: 10.2169/internalmedicine.47.0825)

\section{Introduction}

Chronic kidney disease (CKD) is a major public health problem. On the basis of clinical practice guidelines established by The Japanese Society of Nephrology, 18.7\% of adults in Japan have CKD, which is defined as kidney damage or glomerular filtration rate (GFR) less than $60 \mathrm{~mL} / \mathrm{min} /$ $1.73 \mathrm{~m}^{2}$ for 3 months or more regardless of cause (1), and $4.1 \%$ of these had moderate or severe CKD (2). CKD is associated with an increased risk of cardiovascular disease (CVD) outcomes after adjustment for traditional risk factors $(3,4)$. The development of accelerated, premature atherosclerosis in CKD patients may result from decreased renal function or other non-traditional risk factors.
Arterial stiffness can be systemic or regional, and is an independent predictor for death from CVD in high risk populations (5). Mildly impaired kidney function may contribute to increased arterial stiffness. In a general population without cardiovascular complications, urinary excretion of albumin, which is an early hallmark of renal damage, also indicates the presence of advanced arterial stiffness (6). In hypertensive patients with normal renal function, decreased GFR relates to stiffness of both central elastic and peripheral muscular arteries (7). However, in the general population, it remains unclear whether or not the decline in renal function is an independent risk factor for arterial stiffness.

First, we investigated the relationship between decreased renal function and risk factors such as hypertension, hyperglycemia, and dyslipidemia. Secondly, we investigated de-

${ }^{1}$ Department of Internal Medicine, Nomura Municipal Hospital, Seiyo and ${ }^{2}$ Department of Geriatric Medicine, Ehime University School of Medicine, Touon

Received for publication December 13, 2007; Accepted for publication January 5, 2008

Correspondence to Dr. Ryuichi Kawamoto, rykawamo@ehime.med.or.jp 
Table 1. Characteristics of Various Risk Factors of Subjects Categorized by Estimated Glomerular Filtration Rate

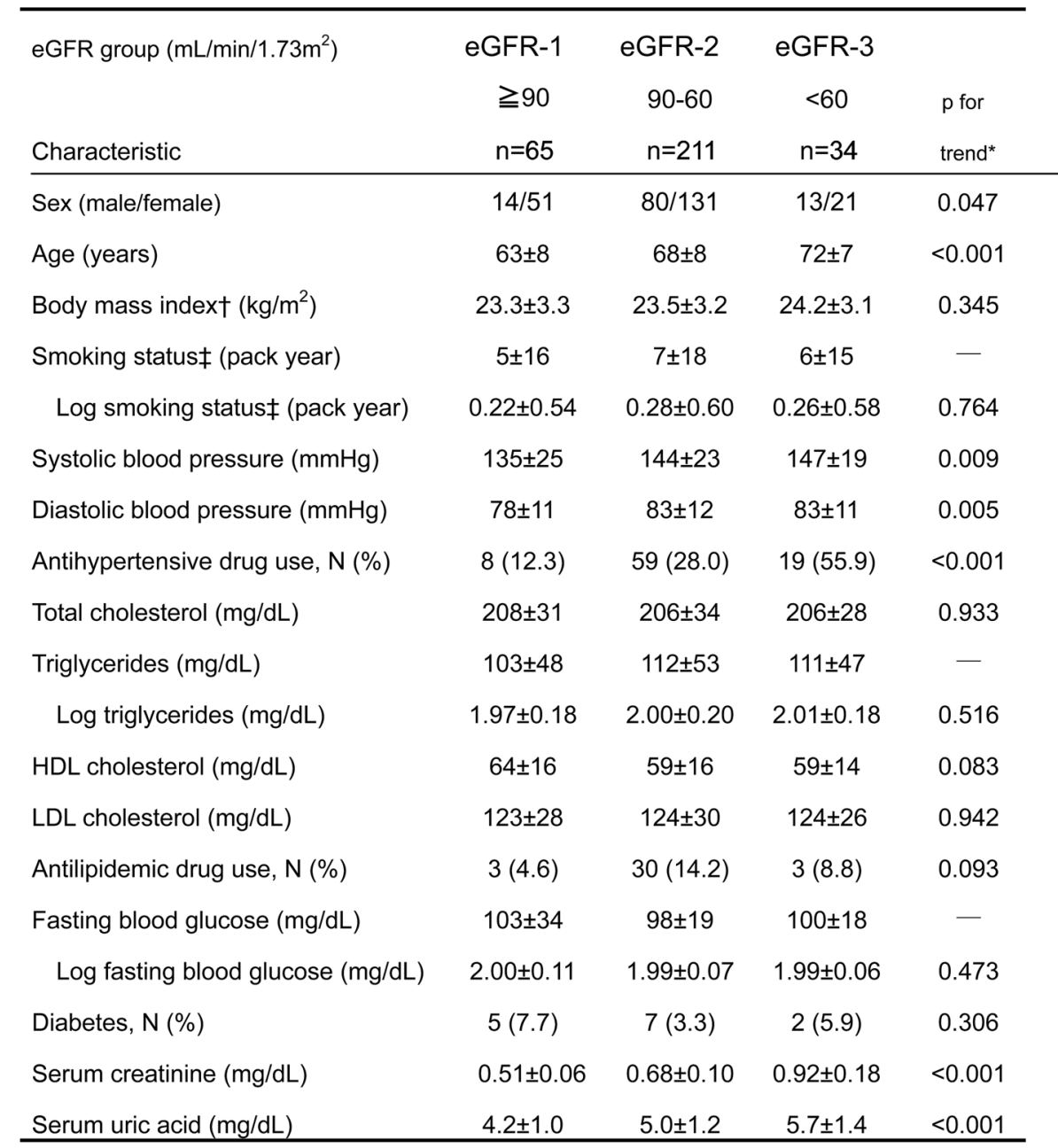

eGFR (estimated glomerular filtration rate) was estimated by using the following equations: $=0.741 \times 175 \times \mathrm{Cr}^{-1.154} \times \mathrm{Age}^{-0.203} \times 0.742($ if female) $\dagger$ Body mass index was calculated using weight in kilograms divided by the square of the height in meters. $¥$ Smoking status: daily consumption (pack) $\times$ duration of smoking (year). HDL, high-density lipoprotein; LDL, low-density lipoprotein.

* One-way ANOVA test or $\chi^{2}$ test.

creased GFR independently associated with arterial stiffness evaluated using pulse wave velocity. For this, we used crosssectional data from community-dwelling subjects free from cardiovascular complications.

\section{Materials and Methods}

\section{Subjects}

Participants residing in Nomura-cho, Seiyo-city were recruited at the time of their annual health examination in 2001. Information on medical history, present conditions, and drugs was obtained by interview. Subjects with a clinical history of stroke, transient ischemic attack, myocardial infarction, or angina were excluded. Informed consent for the procedure was obtained. All procedures were approved by the Ethics Committee of Ehime University School of Medicine.

\section{Evaluation of risk factors}

Information on demographic characteristics and risk factors was collected using clinical files. We measured blood pressure in the right upper arm of patients in a sedentary position using an automatic oscillometric blood pressure recorder (BP-103i; Colin, Aichi, Japan). Cigarette smoking was quantified based on daily consumption and duration of smoking. Total cholesterol (T-C), triglycerides (TG), highdensity lipoprotein cholesterol (HDL-C), fasting blood glucose (FBG), creatinine (enzymatic method), and uric acid were measured during fasting. The low-density lipoprotein cholesterol (LDL-C) level was calculated by the Friedewald formula (8). Patients with TG levels $>400 \mathrm{mg} / \mathrm{dL}$ were ex- 
Table 2. Pulse Wave Velocity of Subjects Categorized by Estimated Glomerular Filtration Rate

\begin{tabular}{lcccc}
\hline eGFR group $\left(\mathrm{mL} / \mathrm{min} / 1.73 \mathrm{~m}^{2}\right)$ & eGFR-1 & eGFR2 & eGFR-3 & \\
& $\geqq 90$ & $90-60$ & $<60$ & $\mathrm{p}$ for \\
Pulse wave velocity & $\mathrm{n}=65$ & $\mathrm{n}=211$ & $\mathrm{n}=34$ & trend $^{*}$ \\
\hline Heart to carotid $(\mathrm{cm} / \mathrm{s})$ & $845.8 \pm 222.5$ & $926.6 \pm 229.6$ & $998.2 \pm 223.1$ & 0.004 \\
Heart to brachial $(\mathrm{cm} / \mathrm{s})$ & $539.7 \pm 174.0$ & $703.9 \pm 266.9$ & $798.3 \pm 373.5$ & $<0.001$ \\
Heart to ankle $(\mathrm{cm} / \mathrm{s})$ & $983.0 \pm 132.7$ & $1088.9 \pm 147.2$ & $1156.2 \pm 198.7$ & $<0.001$ \\
Brachial to ankle $(\mathrm{cm} / \mathrm{s})$ & $1522.7 \pm 289.2$ & $1792.8 \pm 398.9$ & $1954.4 \pm 553.2$ & $<0.001$ \\
Mean pulse wave velocity $(\mathrm{cm} / \mathrm{s})$ & $789.5 \pm 146.8$ & $906.5 \pm 170.2$ & $984.2 \pm 223.9$ & $<0.001$
\end{tabular}

Mean pulse wave velocity was obtained by averaging three pulse wave velocity from heart to carotid, to brachial, and to ankle. * One-way ANOVA test.

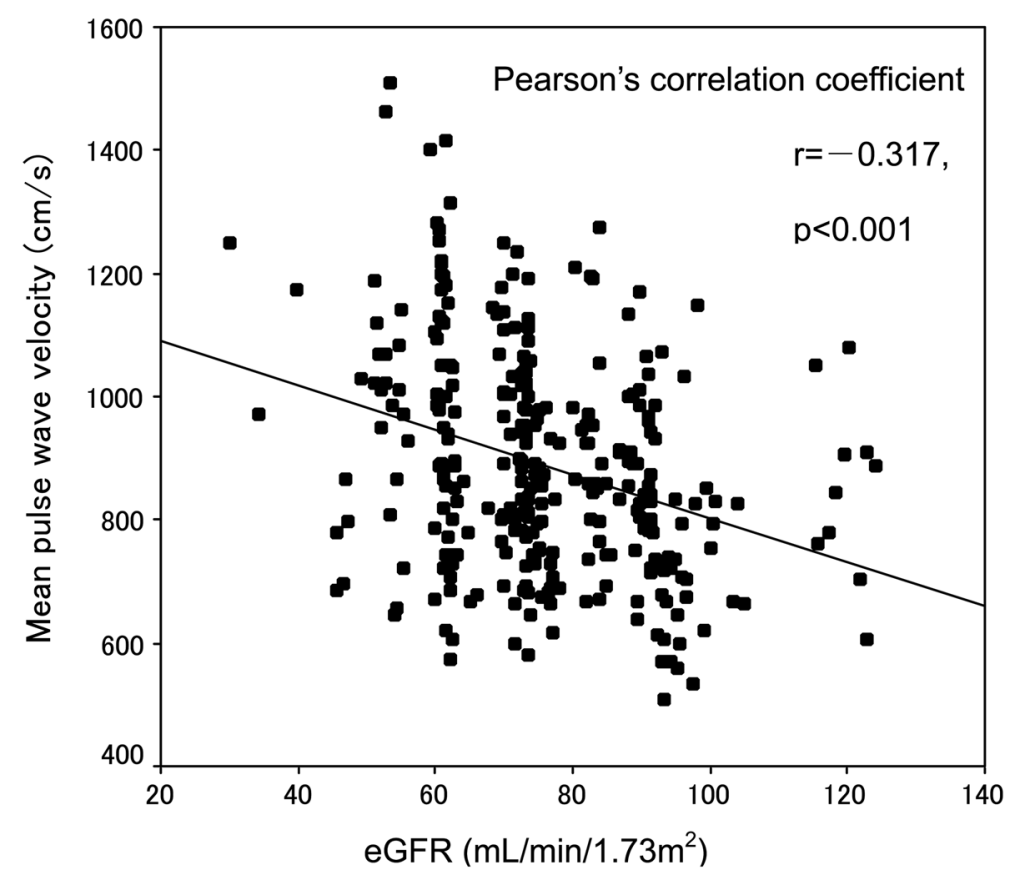

Figure 1. Relationship between estimated glomerular filtration rate (eGFR) and mean pulse wave velocity. eGFR (estimated Glomerular filtration rate) $=0.741 \times 175 \times \mathrm{Cr}^{-1.154} \times \mathrm{Age}^{-0.203} \times 0.742$ (if female).

cluded ( 2 cases). The presence of diabetes mellitus was defined as under medication of antihyperglycemic agents. Glomerular filtration rate was estimated by using the following equation: $\mathrm{eGFR}=0.741 \times 175 \times \mathrm{Cr}^{-1.154} \times \mathrm{Age}^{-0.203} \times 0.742$ (if female) $(9,10)$.

\section{Measurement of pulse wave velocity}

The pulse wave velocity (PWV) was measured using an automatic waveform analyzer (form PWV/Abl; Colin, Co., Ltd., Komaki, Japan). All individuals were examined after resting in the supine position for at least 5 minutes, as described previously (6). Heart to carotid PWV was calculated from measurements of heart and common carotid arterial waveforms, upper limb PWV (heart to brachial) from heart and brachial artery waveforms, and lower limb PWV (heart to ankle) from heart and brachial artery waveforms. We used the mean PWV as a marker of arterial stiffness.

\section{Statistical analysis}

Statistical analysis was performed using SPSS 10.0J (Statistical Package for Social Science, Inc., Chicago, IL, USA). All values are expressed as mean \pm standard deviation, unless otherwise specified. The distribution of smoking status, TG, and FBG were skewed, and therefore log-transformed for analysis. Subjects were divided into three groups based on eGFR (eGFR-1, $\geq 90 \mathrm{~mL} / \mathrm{min} / 1.73 \mathrm{~m}^{2}$; eGFR-2, 60-89 mL/ $\min / 1.73 \mathrm{~m}^{2}$; eGFR-3, 30-59 mL/min/1.73 m²) (11). Differences among the three groups based on eGFR and background parameters were determined by One-way ANOVA or $\chi^{2}$ test. Correlations between various characteristics and mean PWV were determined using Pearson's correlation test. Multiple linear regression analysis was used to evaluate 
Table 3. Relationship between Various Risk Factors Including Estimated Glomerular Filtration Rate and Mean Pulse Wave Velocity

\begin{tabular}{|c|c|c|c|c|c|c|c|c|}
\hline \multirow{3}{*}{ Characteristic } & \multirow{2}{*}{\multicolumn{2}{|c|}{ Pearson's correlation coefficient }} & \multicolumn{6}{|c|}{ Stepwise multiple linear regression analysis } \\
\hline & & & \multicolumn{2}{|c|}{ Model 1} & \multicolumn{2}{|c|}{ Model 2} & \multicolumn{2}{|c|}{ Model 3} \\
\hline & $\mathrm{R}$ & $\mathrm{p}$-value & $\beta$ & $\mathrm{p}$-value & $\beta$ & $\mathrm{p}$-value & $\beta$ & $\mathrm{p}$-value \\
\hline Sex $($ male $=1$, female $=0)$ & 0.044 & 0.437 & - & - & - & - & - & - \\
\hline Age (years) & 0.471 & $<0.001$ & 0.301 & $<0.001$ & 0.275 & $<0.001$ & 0.251 & $<0.001$ \\
\hline Body mass indext $\left(\mathrm{kg} / \mathrm{m}^{2}\right)$ & -0.135 & 0.018 & -0.201 & $<0.001$ & -0.208 & $<0.001$ & -0.228 & $<0.001$ \\
\hline Log smoking statusł (pack year) & -0.065 & 0.253 & - & - & - & - & - & - \\
\hline Systolic blood pressure $(\mathrm{mmHg})$ & 0.562 & $<0.001$ & 0.246 & 0.001 & 0.279 & $<0.001$ & 0.266 & $<0.001$ \\
\hline Diastolic blood pressure $(\mathrm{mmHg})$ & 0.459 & $<0.001$ & 0.244 & $<0.001$ & 0.209 & 0.002 & 0.215 & 0.001 \\
\hline Antihypertensive drug use $(Y e s=1, N o=0)$ & 0.338 & $<0.001$ & 0.185 & $<0.001$ & 0.162 & $<0.001$ & 0.149 & 0.001 \\
\hline Log triglycerides $(\mathrm{mg} / \mathrm{dL})$ & 0.086 & 0.129 & - & - & - & - & - & - \\
\hline HDL cholesterol (mg/dL) & -0.005 & 0.924 & - & - & - & - & - & - \\
\hline LDL cholesterol (mg/dL) & -0.070 & 0.219 & - & - & - & - & - & - \\
\hline Antilipidemic drug use $\left(Y e s=1, N_{0}=0\right)$ & 0.080 & 0.159 & - & - & - & - & - & - \\
\hline Log fasting blood glucose (mg/dL) & 0.095 & 0.094 & - & - & - & - & 0.087 & 0.035 \\
\hline Diabetes $(\mathrm{Yes}=1, \mathrm{No}=0)$ & 0.090 & 0.113 & - & - & - & - & - & - \\
\hline Creatinine (mg/dL) & 0.238 & $<0.001$ & $\mathrm{NI}$ & $\mathrm{NI}$ & 0.133 & 0.002 & $\mathrm{NI}$ & $\mathrm{NI}$ \\
\hline eGFR $\left(\mathrm{mL} / \mathrm{min} / 1.73 \mathrm{~m}^{2}\right) \S$ & -0.317 & $<0.001$ & $\mathrm{NI}$ & $\mathrm{NI}$ & $\mathrm{NI}$ & $\mathrm{NI}$ & -0.171 & $<0.001$ \\
\hline $\mathrm{R}^{2}$ & -..-- & -..-- & 0.481 & $<0.001$ & 0.497 & $<0.001$ & 0.510 & $<0.001$ \\
\hline
\end{tabular}

the contribution of each risk factor, including eGFR, for mean PWV. A value of $\mathrm{p}<0.05$ was considered significant.

\section{Results}

\section{Characteristics of risk factors categorized by eGFR}

One hundred and seven men, aged $68 \pm 9$ (mean \pm standard deviation) years, and 203 women, aged $67 \pm 7$ years completed all the procedures, including PWV measurements. The proportion of men, as well as mean age, systolic blood pressure (SBP), diastolic blood pressure (DBP), antihypertensive drug use, serum creatinine and serum uric acid were higher in the low eGFR group (Table 1). There were no inter-group differences in BMI, smoking status, T-C, log TG, HDL-C, LDL-C, antilipidemic drug use, log FBG, or prevalence of diabetes.

\section{Pulse wave velocity of subjects categorized by eGFR.}

Pulse wave velocities in all sites increased progressively with decreased eGFR (Table 2). Mean PWV increased from the top to bottom GFR group, and eGFR had a significant inverse relation with mean PWV $(\mathrm{r}=-0.317, \mathrm{p}<0.001$; Fig. 1).

\section{Association of eGFR with mean PWV}

Simple correlation coefficients between risk factors and mean PWV are summarized in Table 3. Age, BMI, SBP, DBP, antihypertensive drug use, creatinine and eGFR were significantly correlated with mean PWV (Table 3). To further investigate whether eGFR can improve the prediction of PWV independent of other known risk factors, stepwise multiple linear regression analyses for mean PWV were performed with three models. It revealed that age, BMI, SBP, DBP and antihypertensive drug use were significantly and independently associated with mean PWV (Table 3. model $1)$. Inclusion of creatinine $(\beta=0.133, \mathrm{p}=0.002)$ (mode 2 ) or eGFR $(\beta=-0.171, \mathrm{p}<0.001)$ (model 3$)$ into the model further increased coefficient of determination $\left(\mathrm{R}^{2}\right)$.

\section{Discussion}

To determine the contribution of decreased eGFR to advanced arterial stiffness in the general population, we studied the relationship between cardiovascular risk factors, including eGFR, and arterial stiffness. This study showed that decreased eGFR has an independent, inverse association with arterial stiffness, as reflected by increased heart to carotid, heart to brachial, and heart to ankle PWV. Increased PWV occurred in parallel with the decline in renal function in patients with mild to moderate CKD. In addition, this study confirmed that early-stage $\mathrm{CKD}$, defined as a creatinine-estimated GFR (eGFR) less than $90 \mathrm{~mL} / \mathrm{min} / 1.73$ $\mathrm{m}^{2}(1)$, is very common and significantly associated with higher PWV.

Subjects with mild to moderate CKD had advanced age and hypertension as risk factors. Estimated GFR showed significant associations with blood pressure in addition to PWV, indicating that hypertension was the confounding factor underlying the presence of decreased eGFR. Moreover, the association of CKD with risk for adverse outcomes is strongly related to cardiovascular disease and its risk factors (12). Thus, decreased eGFR may indicate the severity of underlying diseases such as diabetes, hypertension, or atherosclerosis, unrecognized cardiovascular risk factors such as 
dyslipidemia or smoking, or coexistent renal disease and diuretic use. Brachial-ankle PWV is associated with HDL-C, TG, uric acid, FBG, fasting insulin and HBA1c, in addition to SBP and DBP (13), and we found eGFR independently contributed to increased PWV. The present results are in agreement with the hypothesis that mild impairment of renal function might increase arterial stiffness. Schillaci et al (7) found that decreased eGFR was a major determinant of accelerated progression of central and peripheral arterial stiffness in hypertensive patients with normal renal function. Their subjects were about 20 yeas younger than our subjects and their renal function showed eGFR of more than $60 \mathrm{~mL} /$ $\min / 1.73 \mathrm{~m}^{2}$, while $11.3 \%$ of participants in our study had moderate renal dysfunction with eGFR of 30 to $59 \mathrm{~mL} / \mathrm{min} /$ $1.73 \mathrm{~m}^{2}$ and $55.9 \%$ of those were antihypertensive drug users. Thus, our subjects were considered to have been in a more advanced atherosclerotic group.

Diabetic and non-diabetic hypertensive subjects also have higher PWV (14). Insulin resistance is associated with high arterial stiffness and hemodynamic alterations in the common carotid artery $(15,16)$. Insulin resistance causes diabetes, hypertension, metabolic syndrome and renal dysfunction through increased sympathetic stimulation, endothelial dysfunction, aldosterone activation, as well as increased renal sodium absorption, activation of the renin-angiotensionaldosterone system, advanced glycation end products, and lipid peroxidation, resulting in vascular remodeling (17-19). Decreased renal function may increase PWV through insulin resistance. In the present study, hypertension, FBG and metabolic syndrome (by modified criteria for diagnosing MetS in Japan; data not shown) as well as eGFR were independently associated with mean PWV.

Alternatively, decreased renal function may be associated with other non-traditional risk factors not examined in this study, including C-reactive protein (20), albuminuria (21), homocysteine (22), oxidative stress (23), and cytokines (24). Chronic kidney disease may be complicated by both the severity and duration of other causes of CVD, and exacerbate the atherosclerosis caused by CVD risk factors.

Several limitations of this study must be considered. First, based on its cross-sectional study design, the present findings are inherently limited in the ability to eliminate causal relationships between risk factors and PWV. Second, since the majority of the study population had several risk factors, including hypertension and advanced age, we could not eliminate the possible effect of underlying diseases and medications used for hypertension and dyslipidemia on the present findings. Third, formulas for estimating GFR tend to be less accurate in subjects with normal renal function than in patients with CKD (25), although estimated GFR is more accurate than serum creatinine or the Cockcroft-Gault equation (25). Our findings that inclusion of eGFR into the stepwise regression model improved coefficient of determination for mean PWV may further emphasize clinical usefulness of eGFR. Lastly, our definition of eGFR is based on a single assessment of serum creatinine, which may introduce misclassification bias. Therefore the demographics and referral source may limit generalizability.

In conclusion, the present study showed that decreased eGFR is associated with an increased risk of PWV in the general population. The underlying mechanism behind this relationship is unclear, but it seems to be independent from traditional cardiovascular risk factors such as age, BMI, smoking, hypertension, dyslipidemia, and diabetes. Estimation of GFR and conventional risk factors may improve individual risk assessment. Further prospective populationbased studies are needed to investigate the mechanisms underlying this association.

\section{References}

1. Levey AS, Eckardt KU, Tsukamoto Y, et al. Definition and classification of chronic kidney disease: a position statement from Kidney Disease: Improving Global Outcomes (KDIGO). Kidney Int 67: 2089-2100, 2005.

2. Imai E, Horio M, Iseki K, et al. Prevalence of chronic kidney disease (CKD) in the Japanese general population predicted by the MDRD equation modified by a Japanese coefficient. Clin Exp Nephrol 11: 156-163, 2007.

3. Weiner DE, Tighiouart H, Stark PC, et al. Kidney disease as a risk factor for recurrent cardiovascular disease and mortality. Am J Kidney Dis 44: 198-206, 2004.

4. Meisinger C, Doring A, Lowel H. KORA Study Group. Chronic kidney disease and risk of incident myocardial infarction and allcause and cardiovascular disease mortality in middle-aged men and women from the general population. Eur Heart J 27: 12451250, 2006.

5. Pannier B, Guerin AP, Marchais SJ, Guyonvarc'h PM, London GM. Central pulse pressure and mortality in end-stage renal disease. Hypertension 39: 735-738, 2002.

6. Kohara K, Tabara Y, Tachibana R, Nakura J, Miki T. Microalbuminuria and arterial stiffness in a general population: the Shimanami Health Promoting Program (J-SHIPP) study. Hypertens
Res 27: 471-477, 2004.

7. Schillaci G, Pirro M, Mannarino MR, et al. Relation between renal function within the normal range and central and peripheral arterial stiffness in hypertension. Hypertension 48: 616-621, 2006.

8. Friedewald WT, Levy RI, Fredrickson DS. Estimation of the concentration of low-density lipoprotein cholesterol in plasma, without use of the preparative ultracentrifuge. Clin Chem 18: 499-502, 1972.

9. Levey AS, Coresh J, Greene T, et al. Chronic kidney disease epidemiology collaboration. Using standardized serum creatinine values in the modification of diet in renal disease study equation for estimating glomerular filtration rate. Ann Intern Med 145: 247254, 2006.

10. Imai E, Horio M, Nitta $K$, et al. Estimation of glomerular filtration rate by the MDRD study equation modified for Japanese patients with chronic kidney disease. Clin Exp Nephrol 11: 41-50, 2007.

11. National Kidney Foundation. K/DOQI clinical practice guideline to define chronic kidney disease: evaluation, classification and stratification. Am J Kidney Dis 39 [Suppl 2]: S1 -S266, 2002.

12. Culleton BF, Larson MG, Wilson PW, Evans JC, Parfrey PS, Levy D. Cardiovascular disease and mortality in a community-based co- 
hort with mild renal insufficiency. Kidney Int 56: 2214-2219, 1999.

13. Tsubakimoto A, Saito I, Mannami T, et al. Impact of metabolic syndrome on brachial-ankle pulse wave velocity in Japanese. Hypertens Res 29: 29-37, 2006.

14. Kim EJ, Park CG, Park JS, et al. Relationship between blood pressure parameters and pulse wave velocity in normotensive and hypertensive subjects: invasive study. J Hum Hypertens 21: 141148, 2007.

15. Watanabe S, Okura T, Kitami Y, Hiwada K. Carotid hemodynamic alterations in hypertensive patients with insulin resistance. Am J Hypertens 15: 851-856, 2002.

16. Seo HS, Kang TS, Park S, et al. Insulin resistance is associated with arterial stiffness in nondiabetic hypertensives independent of metabolic status. Hypertens Res 28: 945-951, 2005.

17. Sarafidis PA, Lasaridis AN, Nilsson PM, et al. Ambulatory blood pressure reduction after rosiglitazone treatment in patients with type 2 diabetes and hypertension correlates with insulin sensitivity increase. J Hypertens 22: 1769-1777, 2004.

18. Reaven GM, Lithell H, Landsberg L. Hypertension and associated metabolic abnormalities - the role of insulin resistance and the sympathoadrenal system. N Engl J Med 334: 374-381, 1996.

19. Safar ME. Systolic hypertension in the elderly: arterial wall me- chanical properties and the renin-angiotensin-aldosterone system. $\mathbf{J}$ Hypertens 23: 673-681, 2005.

20. Ridker PM, Rifai N, Rose L, Buring JE, Cook NR. Comparison of C-reactive protein and low-density lipoprotein cholesterol levels in the prediction of first cardiovascular events. N Engl J Med 347: 1557-1565, 2002.

21. Gerstein HC, Mann JF, Yi Q, et al. Albuminuria and risk of cardiovascular events, death, and heart failure in diabetic and nondiabetic individuals. JAMA 286: 421-426, 2001.

22. Homocysteine Studies Collaboration. Homocysteine and risk of ischemic heart disease and stroke: a meta-analysis. JAMA 288: 2015-2022, 2002.

23. Drueke T, Witko-Sarsat V, Massy Z, et al. Iron therapy, advanced oxidation protein products, and carotid artery intima-media thickness in end-stage renal disease. Circulation 106: 2212-2217, 2002.

24. Honda H, Qureshi AR, Heimburger O, et al. Serum albumin, Creactive protein, interleukin 6 , and fetuin a as predictors of malnutrition, cardiovascular disease, and mortality in patients with ESRD. Am J Kidney Dis 47: 139-148, 2006.

25. Froissart M, Rossert J, Jacquot C, Paillard M, Houillier P. Predictive performance of the modification of diet in renal disease and Cockcroft-Gault equations for estimating renal function. J Am Soc Nephrol 16: 763-773, 2005.

(C) 2008 The Japanese Society of Internal Medicine http://www.naika.or.jp/imindex.html 